\title{
STEEPEST DESCENT PATHS FOR INTEGRALS DEFINING THE MODIFIED BESSEL FUNCTIONS OF IMAGINARY ORDER
}

\author{
N. M. Temme
}

ABStract. The modified Bessel function of the third kind of purely imaginary order:

$$
K_{i \nu}(x)=\int_{0}^{\infty} e^{-x \cosh t} \cos \nu t d t
$$

is considered for real values of the parameters $\nu, x, x>0$. This function plays an important role in certain problems of mathematical physics. It is the kernel of the Kantorovich-Lebedev transform. In this paper we describe the paths of steepest descent (the saddle point contours) of this integral, giving non-oscillatory integrals. The resulting integral representations are important for obtaining asymptotic expansions and for constructing numerical algorithms. We also consider non-oscillating integrals representing $I_{i \nu}(x)$, the modified Bessel function of the first kind of purely imaginary order. We summarize similar results for the ordinary Bessel functions.

\section{Introduction}

The modified Bessel function of the third kind of purely imaginary order $K_{i \nu}(x)$ is a solution of the differential equation

$$
x^{2} y^{\prime \prime}+x y^{\prime}+\left(\nu^{2}-x^{2}\right) y=0 .
$$

Another solution is the function of the first kind $I_{i \nu}(x)$. Well-known integral representations for these functions are:

$$
K_{i \nu}(x)=\frac{1}{2} \int_{-\infty}^{\infty} e^{-x \cosh t+i \nu t} d t, \quad I_{i \nu}(x)=\frac{1}{2 \pi i} \int_{\infty-\pi i}^{\infty+\pi i} e^{x \cosh t-i \nu t} d t
$$

see Watson [10, p. 181]. We assume that $x>0, \nu \geq 0 . K_{i \nu}(x)$ is real and an even function with respect to $\nu ; I_{i \nu}(x)$ is complex when $\nu>0$. We define

$$
L_{i \nu}(x)=\frac{I_{i \nu}(x)+I_{-i \nu}(x)}{2}
$$

which is a real solution of (1.1), and even with respect to $\nu$. In fact, $L_{i \nu}(x)$ is the real part of $I_{i \nu}(x)$ and $K_{i \nu}(x)$ is the imaginary part of $I_{i \nu}(x)$ (up to a factor)

$$
I_{i \nu}(x)=L_{i \nu}(x)-i \frac{\sinh \pi \nu}{\pi} K_{i \nu}(x) .
$$

Received March 1, 1993, revised June 23, 1993.

Key words and phrases. Modified Bessel function of purely imaginary order, paths of steepest descent, saddle point contours, Kantorovich-Lebedev transform. 
The pairs $\left\{K_{i \nu}(x), I_{i \nu}(x)\right\},\left\{K_{i \nu}(x), L_{i \nu}(x)\right\}$ constitute linearly independent solutions of (1.1), with Wronskians

$$
K_{i \nu}(x) I_{i \nu}^{\prime}(x)-I_{i \nu}(x) K_{i \nu}^{\prime}(x)=K_{i \nu}(x) L_{i \nu}^{\prime}(x)-L_{i \nu}(x) K_{i \nu}^{\prime}(x)=\frac{1}{x}
$$

The function $K_{i \nu}(x)$ plays an important role in potential problems for a wedge. It is the kernel of the Kantorovich-Lebedev transform. We have the pair of transforms

$$
g(y)=\int_{0}^{\infty} f(x) K_{i x}(y) d x, \quad f(x)=\frac{2 x}{\pi^{2}} \sinh (\pi x) \int_{0}^{\infty} y^{-1} K_{i x}(y) g(y) d y
$$

see Lebedev [6] or Sneddon [9]. Asymptotic expansions in connection with this transformation are considered by Naylor [7] and Wong [12].

The functions $K_{i \nu}(x), I_{i \nu}(x)$ also play a role as approximants in uniform asymptotic expansions of solutions of certain second-order linear differential equations; see Dunster [2], [3], [4]. In [2] several properties of the functions are discussed; our function $L_{i \nu}(x)$ is the same as Dunster's, up to a factor depending on $\nu$; in [3] and [4] the results are applied to Legendre functions.

The purpose of this paper is to derive integral representations of $K_{i \nu}(x), I_{i \nu}(x)$ with non-oscillating integrands. Starting points are the integrals in (1.2). The resulting integrals are convenient representations for numerical algorithms, especially when the parameters $x, \nu$ are large. Numerical aspects will be discussed in a later paper.

An extra facet of the paper is giving a demonstration of the method of saddle points. In fact, the integrals considered here are saddle point contour integrals, and they also can be used to derive uniform asymptotic expansions of $K_{i \nu}(x), I_{i \nu}(x)$ for large values of the parameters. In our integral representations we distinguish between the two cases $x \geq \nu$ and $x \leq \nu$. In the second case (especially when the parameters are large), the functions $K_{i \nu}(x), L_{i \nu}(x)$ are rapidly oscillating, and difficult to compute.

In Watson [10, Ch. 8], similar contours for integrals representing the ordinary Bessel functions are discussed. However, the present case of modified Bessel functions of imaginary order is different and more complicated. In particular, the path of steepest descent for $K_{i \nu}(x)$ has rather surprising features when $\nu>x$ (the oscillatory case). For convenience, we summarize the results on non-oscillating integrals for the ordinary Bessel functions in Section 5. In [10] such representations have been used for deriving the Debye type uniform asymptotic expansions of the functions $J_{\nu}(z), Y_{\nu}(z)$ and of the Hankel functions $H_{\nu}^{(1)}(z), H_{\nu}^{(2)}(z)$.

The Debye type uniform asymptotic expansion of $K_{i \nu}(x)$, with $x>\nu$, is given in Erdélyi et al. [5, Vol. II, p. 87]. An Airy type uniform expansion of $K_{i \nu}(x)$ can be found in Balogh [1]; see also Olver [8, p. 425] and [2]. In [2] the expansion of $L_{i \nu}(x)$ is given as well. The Airy type expansions are derived from the differential equation (1.1). The point $x=\nu$ is a so-called turning point of this equation. As mentioned before, the integrals obtained in this paper can be used for obtaining Airy type expansions. When transforming the integrals to standard Airy type form, two saddle points should be taken into account; see Olver [8] or Wong [11] (where also a general introduction to the saddle point method can be found). The remaining saddle points will introduce exponentially small contributions in the asymptotic expansion. 
2. The monotonic case: $x \geq \nu \geq 0$

In this case we write

$$
\nu=x \sin \theta, \quad 0 \leq \theta \leq \frac{1}{2} \pi .
$$

2.1. The contour for $K_{i \nu}(x)$. When $\nu=0$, the real axis is the path of steepest descent of the first integral in (1.2), with a saddle point at $t=0$. When $\nu>0$ the path and saddle point shift upwards in the complex plane. In this case, we write

$$
K_{i \nu}(x)=\frac{1}{2} \int_{-\infty}^{\infty} e^{-\phi(t)} d t, \quad \phi(t)=x(\cosh t-i t \sin \theta) .
$$

The saddle point follows from solving the equation $\phi^{\prime}(t)=0$. It suffices to consider the saddle point $t_{0}=i \theta$. The path of steepest descent is defined by the equation $\Im \phi(t)=\Im \phi\left(t_{0}\right)$. Since $\Im \phi\left(t_{0}\right)=0$, this gives the following relation between the real and imaginary parts of $t$ (we write $t=\tau+i \sigma, t_{0}=\tau_{0}+i \sigma_{0}\left(\right.$ with $\tau_{0}=0, \sigma_{0}=\theta$ ))

$$
\sin \sigma=\sin \theta \frac{\tau}{\sinh \tau}, \quad-\infty<\tau<\infty, \quad 0<\sigma \leq \sigma_{0} \leq \frac{1}{2} \pi .
$$

Hence, the path of steepest descent through the point $t_{0}=i \theta$ is given by

$$
\sigma(\tau)=\arcsin \left(\sin \theta \frac{\tau}{\sinh \tau}\right), \quad-\infty<\tau<\infty
$$

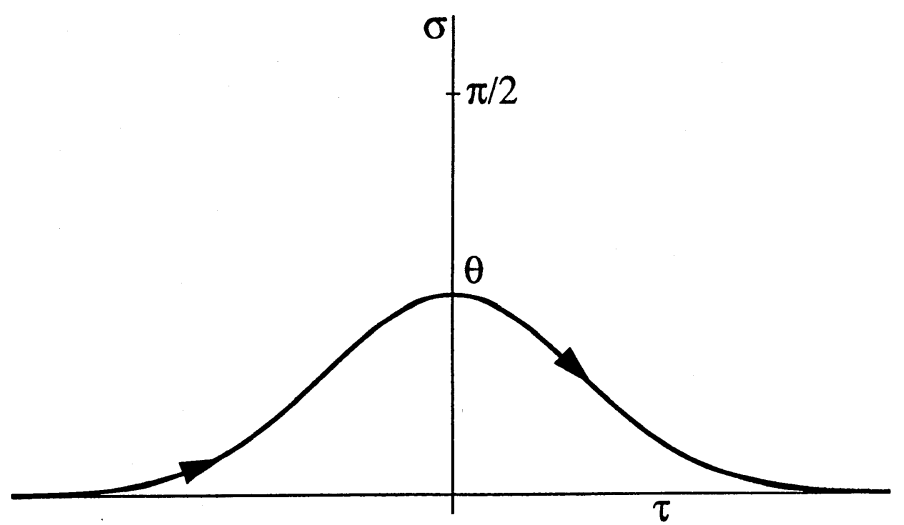

Figure 2.1. Steepest descent path $(2.4), \theta=\frac{\pi}{4}$.

Other solutions of the equation in (2.3) are contours of steepest descent through the saddle points $i( \pm \pi-\theta)$, and are given by

$$
\sigma_{-}(\tau)=-\pi-\sigma(\tau), \quad \sigma_{+}(\tau)=\pi-\sigma(\tau)
$$

These solutions will be used in the next subsection.

Integrating with respect to $\tau$ on the path described by (2.4) gives the representation

$$
K_{i \nu}(x)=\frac{1}{2} \int_{-\infty}^{\infty} e^{-\psi(\tau)} \frac{d t}{d \tau} d \tau, \quad \psi(\tau)=x \cosh \tau \cos \sigma+\nu \sigma
$$


where $\sigma$ as a function of $\tau$ is given in (2.3). The function $\psi(\tau)$ is an even function of $\tau$. Observe that $d t / d \tau=1+i d \sigma / d \tau$ and that $d \sigma / d \tau$, being an odd function of $\tau$, does not give a contribution in (2.6). Hence, we can write

$$
K_{i \nu}(x)=\int_{0}^{\infty} e^{-\psi(\tau)} d \tau, \quad \psi(\tau)=x \cosh \tau \cos \sigma+\nu \sigma
$$

When $x=\nu$ the function $\psi(\tau)$ is not analytic at $\tau=0$. When $x=\nu$, we have, as $\tau \rightarrow 0$ (through real values),

$$
\psi(\tau)=\frac{1}{2} \pi \nu+\sqrt{3} \nu\left|\tau^{3}\right|\left[\frac{4}{27}+\frac{8}{14175} \tau^{4}-\frac{16}{382725} \tau^{6}+\frac{32}{7441875} \tau^{8}+\mathcal{O}\left(\tau^{10}\right)\right] .
$$

From (2.7) it follows that $K_{i \nu}(x)$ is positive when $0 \leq \nu \leq x$. It is convenient to extract the dominant factor, by writing

$$
K_{i \nu}(x)=e^{-\psi(0)} \int_{0}^{\infty} e^{-[\psi(\tau)-\psi(0)]} d \tau
$$

where

$$
\psi(0)=\sqrt{x^{2}-\nu^{2}}+\nu \arcsin \frac{\nu}{x}=x(\cos \theta+\theta \sin \theta) .
$$

2.2. The contour for $I_{i \nu}(x)$ and $L_{i \nu}(x)$. In this case, we need three saddle points. The derivative of the function $\phi(t)=x \cosh t-i \nu t$, occurring in the second integral of (1.2), vanishes at the points

$$
t_{k}=i \sigma_{k}=i\left[(-1)^{k} \theta+k \pi\right], \quad k \in \mathbb{Z} .
$$

The saddle point contour now passes through the saddle points $t_{-1}, t_{0}, t_{1}$, and the path of integration of the second integral in (1.2) is split into three parts: $\mathcal{L}_{-1} \cup \mathcal{L}_{0} \cup \mathcal{L}_{1}$, where

$-\mathcal{L}_{-1}$ runs from $\infty-i \pi$ to $t_{-1}$,

$-\mathcal{L}_{0}$ runs from $t_{-1}$ to $t_{1}$ (a segment of the imaginary axis),

$-\mathcal{L}_{1}$ runs from $t_{1}$ to $\infty+i \pi$.

On each path we have $\Im \phi(t)=0$. On $\mathcal{L}_{ \pm 1}$ the relation between the real and imaginary parts of $t=\tau+i \sigma$ is given by (2.5) with $\tau \geq 0$. Thus we obtain

$$
\begin{aligned}
I_{i \nu}(x)=\frac{1}{2 \pi i} \int_{\mathcal{L}_{-1}} e^{x \cosh \tau \cos \sigma_{-}}+\nu \sigma_{-} & {\left[1+i \frac{d \sigma_{-}}{d \tau}\right] d \tau } \\
+ & \frac{1}{2 \pi} \int_{\sigma_{-1}}^{\sigma_{1}} e^{x \cos \sigma+\nu \sigma} d \sigma \\
& +\frac{1}{2 \pi i} \int_{\mathcal{L}_{1}} e^{x \cosh \tau \cos \sigma_{+}+\nu \sigma_{+}}\left[1+i \frac{d \sigma_{+}}{d \tau}\right] d \tau .
\end{aligned}
$$

Hence, by using (1.4) and separating the real part,

$$
\begin{aligned}
L_{i \nu}(x) & =\frac{1}{2 \pi} \int_{\sigma_{-1}}^{\sigma_{1}} e^{x \cos \sigma+\nu \sigma} d \sigma-\frac{\sinh \pi \nu}{\pi} \int_{0}^{\infty} e^{-\psi(\tau)} \frac{d \sigma}{d \tau} d \tau \\
& =\frac{1}{2 \pi} \int_{\sigma_{-1}}^{\sigma_{1}} e^{x \cos \sigma+\nu \sigma} d \sigma-\frac{\sinh \pi \nu}{\pi} \int_{0}^{\sigma_{0}} e^{-\psi(\tau)} d \sigma
\end{aligned}
$$




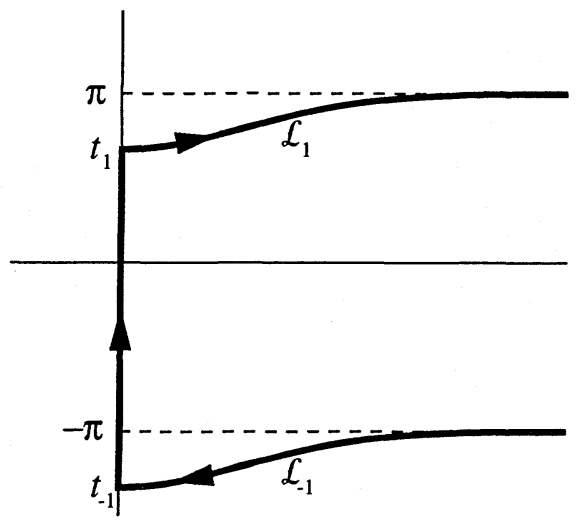

FIGURE 2.2. Steepest descent path used in (2.10)

where $x \geq \nu \geq 0, \psi(\tau)$ is given in (2.7), the relation between $\tau$ and $\sigma$ in (2.3), and

$$
\frac{d \sigma}{d \tau}=\tan \sigma\left(\frac{1}{\tau}-\operatorname{coth} \tau\right)
$$

This gives the requested representation of $I_{i \nu}(x)$ in terms of non-oscillating integrals. The integral on the interval $\left[\sigma_{-1}, \sigma_{1}\right]$ gives the main contribution, especially when the parameters are large. The integrand peaks at the point $\sigma=\sigma_{0}=\theta$. Hence, the main contribution to this integral comes from a small neighborhood of this point. At this point the integrand assumes the value $\exp [\psi(0)]$, compare (2.8).

\section{The oscillatory case: $0<x \leq \nu$}

Under the present condition, we write

$$
\nu=x \cosh \mu, \quad \mu>0 .
$$

3.1. The contour for $K_{i \nu}(x)$. In this case, an infinite number of saddle points are used for obtaining the steepest descent path. However, a simple summation procedure reduces the number of saddle points to a few, as in the case of $I_{i \nu}(x)$ in the previous section.

We write $\phi(t)$ of $(2.2)$ in the form $\phi(t)=x(\cosh t-i t \cosh \mu)$. It follows that the saddle points of the integrands in (1.2) are now given by

$$
t_{k}^{ \pm}= \pm \tau_{0}+\frac{1}{2} \pi i+2 k \pi i, \quad \tau_{0}=\mu, \quad k \in \mathbb{Z}
$$

The saddle point contour through the saddle $t_{k}^{ \pm}$is defined by the equation $\Im \phi(t)=$ $\Im \phi\left(t_{k}^{ \pm}\right)= \pm x(\sinh \mu-\mu \cosh \mu)$, that is, by

$$
\sin \sigma=\cosh \mu \frac{\tau}{\sinh \tau} \pm \frac{\sinh \mu-\mu \cosh \mu}{\sinh \tau}
$$

which is independent of $k$. The contours cannot have common points with the imaginary axis (where $\tau=0$ ). In fact, this axis separates two groups of contours, namely, those through $t_{k}^{+}$from those through $t_{k}^{-}$, the first group corresponding to positive values of $\tau$, and the second one to negative values. The parabola shaped curves satisfying (3.2) are shown in Figure 3.1. On each 'parabola', two saddle points are located. 
Hence, a complete parabola cannot serve as a path of descent, since one branch extending to infinity is always a path of ascent. Only parts of the parabola shaped contours are used for the steepest descent paths.

First, the original contour in (1.2) for $K_{i \nu}(x)$ is split into two parts: $\mathcal{L}^{-} \cup \mathcal{L}^{+}$, where

$-\mathcal{L}^{-}$runs from $-\infty$ to 0 and from 0 to $+i \infty$,

$-\mathcal{L}^{+}$runs from $+i \infty$ to 0 and from 0 to $+\infty$.

Since $\nu>0$, the integrals along $\mathcal{L}^{ \pm}$are convergent at $+i \infty$. Next, we deform $\mathcal{L}^{ \pm}$ along the thickened parts of the saddle point contours shown in Figure 3.1. Owing to the symmetry, we can consider $\mathcal{L}^{+}$only, along which we take twice the real part of this integral. On the lower branch of $\mathcal{L}^{+}$(running from $t_{0}^{+}$to $+\infty$ ), we integrate with respect to $\tau$, on the upper branch (running from $+i \infty$ to $t_{0}^{+}$), we integrate with respect to $\sigma$. The result is

$$
K_{i \nu}(x)=\Re\left\{e^{-i \chi}\left[\int_{\tau_{0}}^{\infty} e^{-\psi(\tau)}\left(1+i \frac{d \sigma}{d \tau}\right) d \tau-\int_{\frac{1}{2} \pi}^{\infty} e^{-\psi(\tau)}\left(\frac{d \tau}{d \sigma}+i\right) d \sigma\right]\right\},
$$

where

$$
\chi=\Im \phi\left(t_{0}^{+}\right)=x \sinh \tau_{0}-\nu \tau_{0}, \quad e^{-\psi(\tau)}=e^{-x \cosh \tau \cos \sigma-\nu \sigma},
$$

and the relation between $\tau$ and $\sigma$ is given in (3.2) (with the + sign).

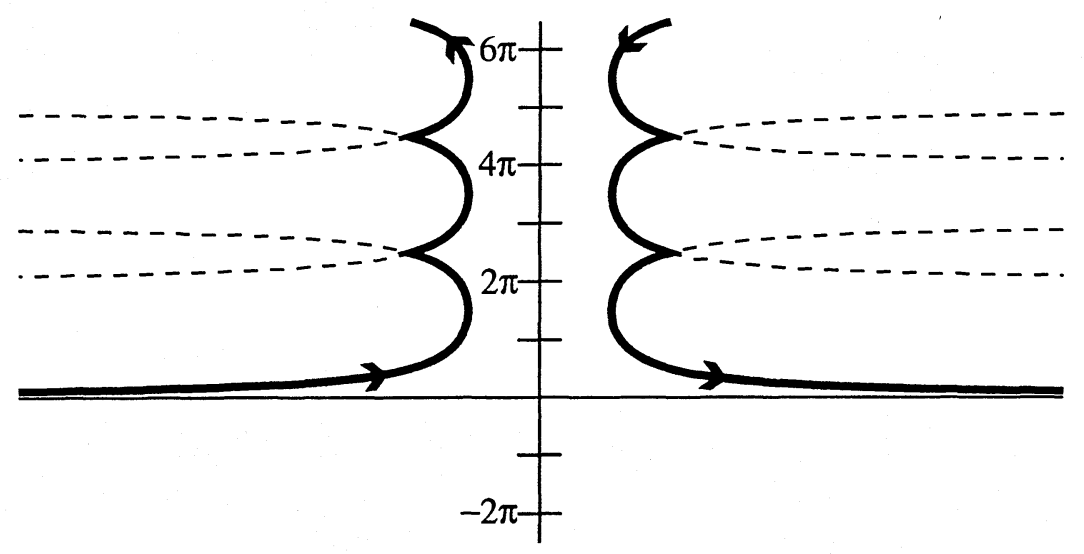

FIGURE 3.3. Steepest descent path used in (3.3)

The second integral in (3.3) can be reduced to an integral over a finite interval. The fact is that the function

$$
P(\sigma)=e^{-x \cosh \tau \cos \sigma}\left(\frac{d \tau}{d \sigma}+i\right)
$$

is periodic with respect to $\sigma$ with period $2 \pi, P(\sigma)=P(\sigma+2 \pi)$. It follows that

$$
\int_{\frac{1}{2} \pi}^{\infty} P(\sigma) e^{-\nu \sigma} d \sigma=\frac{1}{1-e^{-2 \pi \nu}} \int_{\frac{1}{2} \pi}^{\frac{5}{2} \pi} P(\sigma) e^{-\nu \sigma} d \sigma
$$


Thus we obtain the representation

$$
\begin{aligned}
K_{i \nu}(x)=\cos \chi & {\left[\int_{\tau_{0}}^{\infty} e^{-\psi(\tau)} d \tau-\frac{1}{1-e^{-2 \pi \nu}} \int_{\frac{1}{2} \pi}^{\frac{5}{2} \pi} e^{-\psi(\tau)} \frac{d \tau}{d \sigma} d \sigma\right] } \\
& +\sin \chi\left[\int_{\tau_{0}}^{\infty} e^{-\psi(\tau)} \frac{d \sigma}{d \tau} d \tau-\frac{1}{1-e^{-2 \pi \nu}} \int_{\frac{1}{2} \pi}^{\frac{5}{2} \pi} e^{-\psi(\tau)} d \sigma\right]
\end{aligned}
$$

When the parameters $x, \nu$ are large the main contribution comes from the point $t_{0}^{+}=$ $\tau_{0}+\pi i / 2$. At this point, we have $\exp \left[-\psi\left(\tau_{0}\right)\right]=\exp (-\pi \nu / 2)$. This quantity gives a proper estimate of the maximal absolute value of the oscillating function $K_{i \nu}(x)$.

REMARK 3.1. Integrals with respect to $\tau$ may be replaced by integrals with respect to $\sigma$; for instance,

$$
\int_{\tau_{0}}^{\infty} e^{-\psi(\tau)} \frac{d \sigma}{d \tau} d \tau=\int_{0}^{\frac{1}{2} \pi} e^{-\psi(\tau)} d \sigma
$$

In a numerical sense, the integrals with respect to $\tau$ are simpler than those with respect to $\sigma$. The reason is that a simple inversion of the sine function in (3.2) gives an explicit representation of $\sigma(\tau)$. Such a simple inversion for $\tau(\sigma)$ is not available.

REMARK 3.2. Another point is that if, for instance, one wants to replace

$$
\int_{\pi / 2}^{5 \pi / 2} e^{-\psi(\tau)} \frac{d \tau}{d \sigma} d \sigma
$$

by an integral with respect to $\tau$, one has to write first

$$
\int_{\pi / 2}^{5 \pi / 2} e^{-\psi(\tau)} \frac{d \tau}{d \sigma} d \sigma=\int_{\pi / 2}^{3 \pi / 2} e^{-\psi(\tau)} \frac{d \tau}{d \sigma} d \sigma+\int_{3 \pi / 2}^{5 \pi / 2} e^{-\psi(\tau)} \frac{d \tau}{d \sigma} d \sigma
$$

since $\sigma(\tau)$ is two-valued on the corresponding $\tau$-interval.

3.2. The contour for $I_{i \nu}(x)$ and $L_{i \nu}(x)$. The second integral in (1.2) can be split up into three parts:

$-\mathcal{L}_{-1}$ runs from $\infty-\pi i$ to $t_{-1}^{+}$,

$-\mathcal{L}_{0}$ runs from $t_{-1}^{+}$to $t_{0}^{+}$,

$-\mathcal{L}_{1}$ runs from $t_{0}^{+}$to $\infty+\pi i$.

The integrals along $\mathcal{L}_{-1}$ and $\mathcal{L}_{1}$ are transformed to integrals along the path from $t_{0}^{+}$to $\infty$, which has been used for $K_{i \nu}(x)$. This is done by changing $\sigma$ into $-\pi-$ $\sigma, \pi-\sigma$, respectively. The integral along $\mathcal{L}_{0}$ is shifted upwards by changing $\sigma$ into $-2 \pi+\sigma$; afterwards we reverse the direction of integration from $\pi / 2$ to $5 \pi / 2$ into $5 \pi / 2$ to $\pi / 2$. The two operations for $\mathcal{L}_{0}$ can be established by the single transformation $\sigma \rightarrow \pi-\sigma$. In this way we obtain integrals as used for $K_{i \nu}(x)$. Corresponding $\tau$ values are not changed during these transformations, as follows from the relation (3.2). Corresponding values of $d \sigma / d \tau$ do change sign, however.

So we obtain

$$
\begin{aligned}
I_{i \nu}(x)=\frac{e^{i \chi}}{2 \pi i}\left[\left(e^{\pi \nu}-e^{-\pi \nu}\right) \int_{\tau_{0}}^{\infty} e^{-\psi(\tau)}(1\right. & \left.-i \frac{d \sigma}{d \tau}\right) d \tau \\
& \left.+e^{\pi \nu} \int_{\frac{1}{2} \pi}^{\frac{5}{2} \pi} e^{-\psi(\tau)}\left(-\frac{d \tau}{d \sigma}+i\right) d \sigma\right] .
\end{aligned}
$$




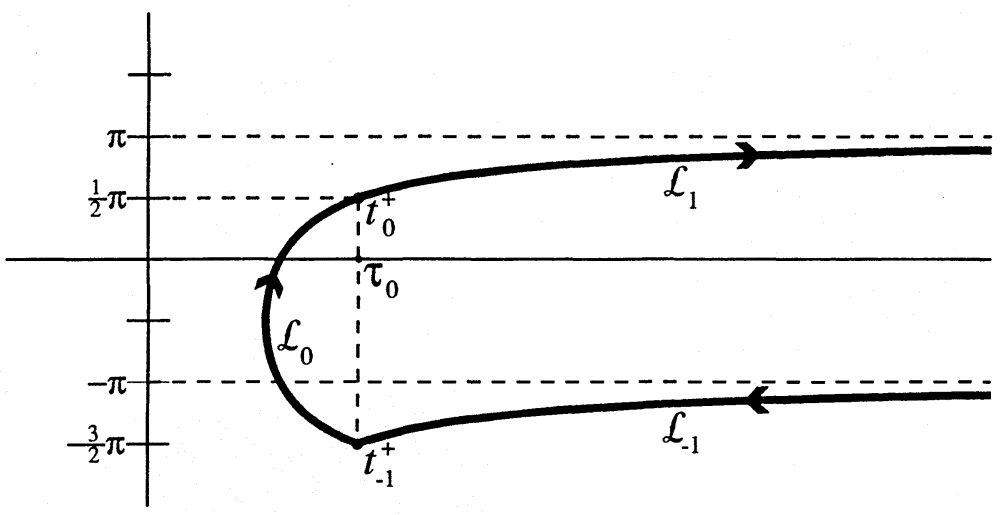

Figure 3.4. Steepest descent path used in (3.6)

The quantities $\chi$ and $e^{-\psi(\tau)}$ are given in (3.4). The imaginary part of $I_{i \nu}(x)$ corresponds to (3.5) (see also (1.4)). Separating the real part gives

$$
\begin{aligned}
L_{i \nu}(x)= & \frac{\cos \chi}{2 \pi}\left[-2 \sinh \pi \nu \int_{\tau_{0}}^{\infty} e^{-\psi(\tau)} \frac{d \sigma}{d \tau} d \tau+e^{\pi \nu} \int_{\frac{1}{2} \pi}^{\frac{5}{2} \pi} e^{-\psi(\tau)} d \sigma\right] \\
& +\frac{\sin \chi}{2 \pi}\left[2 \sinh \pi \nu \int_{\tau_{0}}^{\infty} e^{-\psi(\tau)} d \tau-e^{\pi \nu} \int_{\frac{1}{2} \pi}^{\frac{5}{2} \pi} e^{-\psi(\tau)} \frac{d \tau}{d \sigma} d \sigma\right]
\end{aligned}
$$

When the parameters $x, \nu$ are large, the main contribution again comes from the point $t_{0}^{+}=\tau_{0}+\frac{1}{2} \pi i$. At this point we have $\exp \left[-\psi\left(\tau_{0}\right)\right]=\exp \left(-\frac{1}{2} \pi \nu\right)$. Hence, the quantity $\exp \left(\frac{1}{2} \pi \nu\right)$ gives a proper estimate of the maximal absolute value of the oscillating functions $I_{i \nu}(x)$ and $L_{i \nu}(x)$.

\section{Results for the derivatives}

It is also of interest to have results for the derivatives; they follow rather straightforwardly from the earlier methods.

4.1. The monotonic case: $\nu=x \sin \theta$. The derivatives of $K_{i \nu}(x), I_{i \nu}(x)$ are obtained from (1.2). We have

$$
\begin{aligned}
K_{i \nu}^{\prime}(x) & =-\frac{1}{2} \int_{-\infty}^{\infty} \cosh t e^{-x \cosh t+i \nu t} d t \\
I_{i \nu}^{\prime}(x) & =\frac{1}{2 \pi i} \int_{\infty-\pi i}^{\infty+\pi i} \cosh t e^{x \cosh t-i \nu t} d t .
\end{aligned}
$$

The analogue of (2.6) reads

$$
K_{i \nu}^{\prime}(x)=\frac{1}{2} \int_{-\infty}^{\infty} e^{-\psi(\tau)} g(\tau) d \tau, \quad g(\tau)=\frac{\sin \theta \sin \sigma-\cosh \tau}{\cos \sigma}
$$

the relation between $\tau$ and $\sigma$ given by (2.4). The function $g(\tau)$ is the real part of $-\cosh t d t / d \tau$; the imaginary part is an odd function of $\tau$ and does not give contributions. 
The result for the $I$-function is given in terms of the $L$-function. We obtain from (1.4)

$$
I_{i \nu}^{\prime}(x)=L_{i \nu}^{\prime}(x)-i \frac{\sinh \pi \nu}{\pi} K_{i \nu}^{\prime}(x)
$$

with $L_{i \nu}^{\prime}(x)$ given as the analogue of (2.11) by

$$
L_{i \nu}^{\prime}(x)=\frac{1}{2 \pi} \int_{\sigma_{-1}}^{\sigma_{1}} \cos \sigma e^{x \cos \sigma+\nu \sigma} d \sigma+\frac{\sinh \pi \nu}{\pi} \int_{0}^{\infty} e^{-\psi(\tau)} h(\tau) d \tau
$$

where

$$
h(\tau)=\sin \sigma\left(\frac{\cosh \tau}{\tau}-\frac{1}{\sinh \tau}\right)
$$

This function is obtained by taking the real part of

$$
\frac{1}{2 \pi i}\left[-\cosh \left(\tau+i \sigma_{-}\right) e^{-\pi \nu}\left(1+i \frac{d \sigma_{-}}{d \tau}\right)+\cosh \left(\tau+i \sigma_{+}\right) e^{+\pi \nu}\left(1+i \frac{d \sigma_{+}}{d \tau}\right)\right]
$$

where $\sigma_{ \pm}$are given in (2.5), $\sigma$ in (2.4), and $d \sigma_{ \pm} / d \tau=-d \sigma / d \tau$ in (2.12).

4.2. The oscillatory case: $\nu=x \cosh \mu$. Following the analysis that leads to (3.5), we obtain

$$
\begin{aligned}
& K_{i \nu}^{\prime}(x)=\cos \chi\left[\int_{\tau_{0}}^{\infty} e^{-\psi(\tau)} A(\tau) d \tau-\frac{1}{1-e^{-2 \pi \nu}} \int_{\frac{1}{2} \pi}^{\frac{5}{2} \pi} e^{-\psi(\tau)} B(\tau) d \sigma\right] \\
& \quad+\sin \chi\left[\int_{\tau_{0}}^{\infty} e^{-\psi(\tau)} C(\tau) d \tau-\frac{1}{1-e^{-2 \pi \nu}} \int_{\frac{1}{2} \pi}^{\frac{5}{2} \pi} e^{-\psi(\tau)} D(\tau) d \sigma\right]
\end{aligned}
$$

where

$$
\begin{aligned}
& A(\tau)=-\cosh \tau \cos \sigma+\sinh \tau \sin \sigma \frac{d \sigma}{d \tau}, \quad B(\tau)=A(\tau) \frac{d \tau}{d \sigma} \\
& C(\tau)=-\sinh \tau \sin \sigma-\cosh \tau \cos \sigma \frac{d \sigma}{d \tau}, \quad D(\tau)=C(\tau) \frac{d \tau}{d \sigma}
\end{aligned}
$$

and the relation between $\tau$ and $\sigma$ is given in (3.1) (with the + sign).

For the function $L_{i \nu}^{\prime}(x)$, we obtain

$$
\begin{aligned}
L_{i \nu}^{\prime}(x) & =\frac{\cos \chi}{2 \pi}\left[-2 \sinh \pi \nu \int_{\tau_{0}}^{\infty} e^{-\psi(\tau)} C(\tau) d \tau+e^{\pi \nu} \int_{\frac{1}{2} \pi}^{\frac{5}{2} \pi} e^{-\psi(\tau)} D(\tau) d \sigma\right] \\
& +\frac{\sin \chi}{2 \pi}\left[-2 \sinh \pi \nu \int_{\tau_{0}}^{\infty} e^{-\psi(\tau)} A(\tau) d \tau+e^{\pi \nu} \int_{\frac{1}{2} \pi}^{\frac{5}{2} \pi} e^{-\psi(\tau)} B(\tau) d \sigma\right]
\end{aligned}
$$




\section{Non-oscillating integrals for the ordinary Bessel functions}

We give similar integrals for the ordinary Bessel functions. Some of the results can be found in Watson [10, Ch. 8]. We only consider real values of the parameters of the Bessel functions. The starting points are the contour integrals for the Hankel functions given by

$$
H_{\nu}^{(1)}(x)=\frac{1}{\pi i} \int_{-\infty}^{\infty+i \pi} e^{x \sinh t-\nu t} d t, \quad H_{\nu}^{(2)}(x)=\frac{-1}{\pi i} \int_{-\infty}^{\infty-i \pi} e^{x \sinh t-\nu t} d t .
$$

Furthermore we use the relations

$$
J_{\nu}(x)=\frac{1}{2}\left[H_{\nu}^{(1)}(x)+H_{\nu}^{(2)}(x)\right], \quad Y_{\nu}(x)=\frac{1}{2 i}\left[H_{\nu}^{(1)}(x)-H_{\nu}^{(2)}(x)\right] .
$$

5.1. The monotonic case: $\nu=x \cosh \mu, \mu \geq 0$. In this case, we concentrate on the functions $J_{\nu}(x), Y_{\nu}(x)$. For the first function, we have

$$
J_{\nu}(x)=\frac{1}{2 \pi i} \int_{\infty-i \pi}^{\infty+i \pi} e^{x \sinh t-\nu t} d t
$$

The real saddle points of the function $\phi(t)=x \sinh t-\nu t=x(\sinh t-t \cosh \mu)$ are located at $t= \pm \mu$. The path of steepest descent through $t=\mu$ is defined by (we write $t=\tau+i \sigma)$

$$
\tau=\operatorname{arccosh}\left(\cosh \mu \frac{\sigma}{\sin \sigma}\right), \quad-\pi<\sigma<\pi .
$$

Using this relation, we obtain the representation

$$
J_{\nu}(x)=\frac{1}{2 \pi} \int_{-\pi}^{\pi} e^{x(\sinh \tau \cos \sigma-\tau \cosh \mu)} d \sigma .
$$

For the Neumann function, we use both saddle points and obtain

$$
Y_{\nu}(x)=-\frac{1}{\pi} \int_{-\infty}^{\mu} e^{x(\sinh t-t \cosh \mu)} d t-\frac{1}{\pi} \int_{0}^{\pi} e^{x(\sinh \tau \cos \sigma-\tau \cosh \mu)} \frac{d \tau}{d \sigma} d \sigma .
$$

The main contribution in the first result comes from the point $\sigma=0$, which corresponds to the saddle point $t=\mu$; the main contribution for the Neumann function comes from the saddle point $t=-\mu$.

5.2. The oscillatory case: $\nu=x \cos \theta, 0 \leq \theta \leq \frac{1}{2} \pi$. It is now convenient to consider the Hankel functions. The saddle point of interest for the first Hankel function is $t=i \theta$, for the second function, the point is $t=-i \theta$. After standard manipulations, we obtain

$$
H_{\nu}^{(1)}(x)=\frac{e^{i \chi}}{\pi} \int_{0}^{\pi} e^{x(\sinh \tau \cos \sigma-\tau \cos \theta)}\left(1-i \frac{d \tau}{d \sigma}\right) d \sigma
$$

where

$$
\chi=x(\sin \theta-\theta \cos \theta) \text {. }
$$

The relation between $\tau$ and $\sigma$ is defined by

$$
\tau=\operatorname{arccosh} \frac{\sigma \cos \theta+\sin \theta-\theta \cos \theta}{\sin \sigma}, \quad 0<\sigma<\pi .
$$


The sign of $\tau$ is positive when $\sigma>\theta$ and negative when $\sigma<\theta$. For the second Hankel function, we have

$$
H_{\nu}^{(2)}(x)=\frac{e^{-i \chi}}{\pi} \int_{0}^{\pi} e^{x(\sinh \tau \cos \sigma-\tau \cos \theta)}\left(1+i \frac{d \tau}{d \sigma}\right) d \sigma .
$$

As usual, the result for one Hankel function follows from the other one by changing the sign of the imaginary unit $i$.

In this case it is quite easy to derive the corresponding results for the derivatives of the functions.

Acknowledgment. The author wishes to thank Dr. Adri Olde Daalhuis for his help with making the pictures.

\section{References}

1. C. B. Balogh, Asymptotic expansions of the modified Bessel functions of the third kind of imaginary order, SIAM J. Appl. Math. 15 (1967), 1315-1323.

2. T. M. Dunster, Bessel functions of purely imaginary order, with an application to second-order linear differential equations having a large parameter, SIAM J. Math. Anal. 21 (1990), 9951018.

3. - Uniform asymptotic solutions of second-order linear differential equations having a double pole with complex exponent and a coalescing turning point, SIAM J. Math. Anal. 21 (1990), 1594-1618.

4. - Conical functions with one or both parameters large, Proc. Roy. Soc. Edinburgh 119A (1991), 311-327.

5. A. Erdélyi, W. Magnus, F. Oberhettinger, and F. Tricomi, Higher Transcendental Functions, Vols. I, II, III, Bateman Manuscript Project, McGraw-Hill, New York, 1953.

6. N. N. Lebedev, Special Functions and Their Applications, Prentice-Hall, Englewood Cliffs, New Jersey, 1965.

7. D. Naylor, On an asymptotic expansion of the Kantorovich-Lebedev transform, Applicable Analysis 39 (1967), 249-263.

8. F. W. J. Olver, Asymptotics and Special Functions, Academic Press, New York, 1974.

9. I. N. Sneddon, The Use of Integral Transforms, McGraw-Hill, New York, 1972.

10. G. N. Watson, A Treatise on the Theory of Bessel Functions, Cambridge Univ. Press, London, 1944.

11. R. Wong, Asymptotic Approximations of Integrals, Academic Press, New York, 1989.

12. - Asymptotic expansions of the Kantorovich-Lebedev transform, Applicable Analysis 12 (1981), 161-172.

CWI, P. O. Box 94079, 1090GB, 1009 AB Amsterdam, The Netherlands

E-mail address: nicot@cwi.nl 